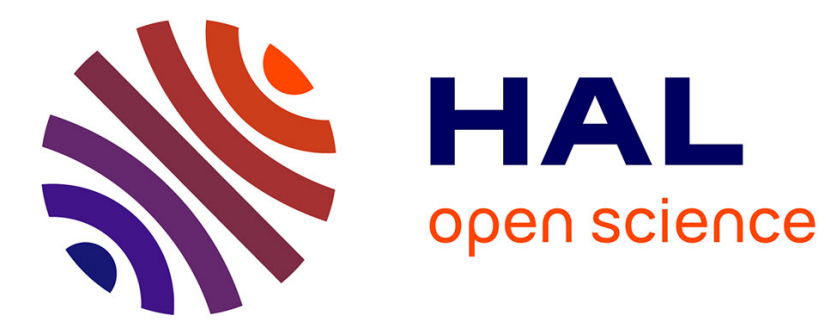

\title{
Manipulation of flexible objects
}

Andrea Cherubini

\section{To cite this version:}

Andrea Cherubini. Manipulation of flexible objects. 2020. hal-02518483

\section{HAL Id: hal-02518483 \\ https://hal.science/hal-02518483}

Preprint submitted on 25 Mar 2020

HAL is a multi-disciplinary open access archive for the deposit and dissemination of scientific research documents, whether they are published or not. The documents may come from teaching and research institutions in France or abroad, or from public or private research centers.
L'archive ouverte pluridisciplinaire HAL, est destinée au dépôt et à la diffusion de documents scientifiques de niveau recherche, publiés ou non, émanant des établissements d'enseignement et de recherche français ou étrangers, des laboratoires publics ou privés. 


\title{
Manipulation of flexible objects
}

\author{
Andrea Cherubini
}

Received: date / Accepted: date

\begin{abstract}
Since decades, industrial robots are capable of grasping and manipulating rigid objects. This is possible because the geometry and position of such objects is known beforehand by the control software. For service robots, the task is more challenging, since it requires the robot to know all types of objects present in the environment (e.g., in an apartment) and to recognize them on the fly to automatically determine the manipulation strategy. Manipulating flexible objects is even more difficult, since along with their position, their shape also varies depending on the applied forces and on their physical properties. Yet, this task is paramount in many fields, spanning from construction to food industry and surgery. This essay reviews applications, challenges, past research works and perspectives of flexible robot manipulation.
\end{abstract}

Keywords Robot manipulation · Control

PACS 02.30.Yy Control theory

Mathematics Subject Classification (2010) 68T40 Robotics · 70 E60 Robot dynamics and control . 93C85 Automated systems · 70Q05 Control of mechanical systems · 58E25 Applications to control theory · 93B07 Observability

\section{Examples of Application}

The first robots were designed for the industrial sector. Yet (Market Research Engine, 2018) forecasts that by 2022 the service robotics market will reach almost 24 billion $\$$, with a compound annual growth rate of more than $15 \%$. This market includes personal service robots for automating household tasks (e.g., cleaning or cooking) and professional service robots (e.g., for construction, farming or medical tasks).

To fulfill such huge demand, robots must be capable of efficiently operating in new environments, which are not as predictable and customized as industrial ones.

A. Cherubini

LIRMM, Université de Montpellier, CNRS, Montpellier, France. E-mail: cherubini@lirmm.fr 

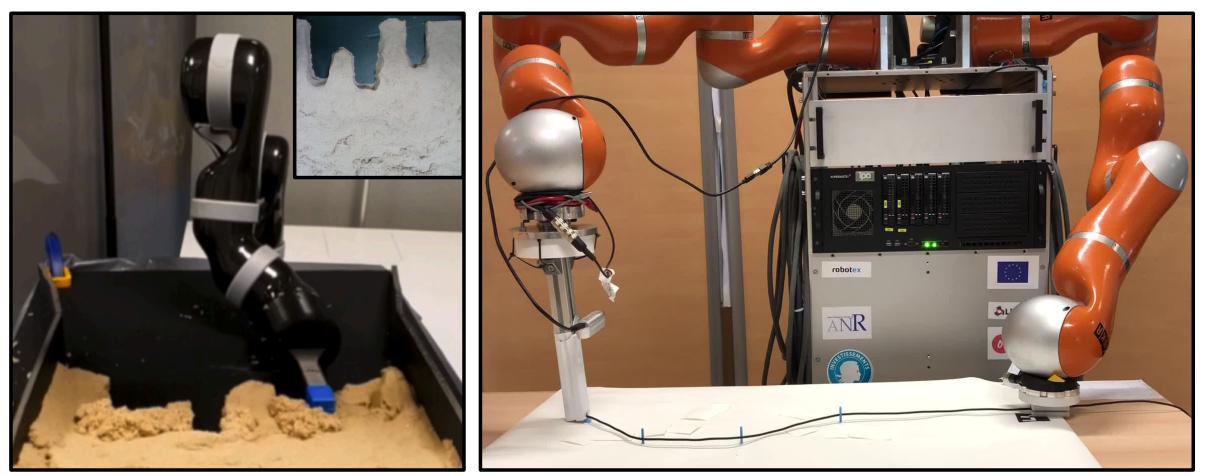

Fig. 1 Two examples of manipulation of flexible materials. Left (Cherubini, 2017): a robot pushes some granular material (kinetic sand) to give it a desired shape, shown in the top right. Right (Zhu et al., 2020): a dual arm robot exploits physical contacts with the environment to position a cable for harnessing.

Manipulating flexible objects is a typical requirement of many service robotic scenarios. For instance, robots will have to shape granular materials for construction and food preparation: they will have to mold clay or plaster as artisans, and dough as bakers. In the house of the future, they will have to iron and fold clothes and to look after the garden, by moving soil and carefully picking fruits of different hardness. Professional service robots operating in farms must also be capable of the latter tasks. Soft/plastic matter is very common in the healthcare field. Surgical robots should interact safely with human organs and tissue. The same applies to robots designed for paramedic tasks, such as drug injection or massage.

While the above paragraph should have convinced the reader of the importance of soft manipulation in service robots, it is noteworthy that the manufacturing industry has also recently turned its interest towards such problem. Soon, industrial robots will have to automatically shape wires and metal sheets, and to insert cables, gaskets and pipes - all of which are non-rigid and cannot be handled by classic robot controllers. Figure 1 illustrates two applications, with robots shaping granular matter (left) and positioning a cable for harnessing (right).

Despite this broad range of applications, robotic manipulation of flexible objects is still an open research field. The next section outlines the main challenges to be addressed within this fascinating problem.

\section{Challenges}

(Cambridge Dictionary, 2019) defines as flexible object one which "can be bent easily without breaking". External forces applied to it will deform the object, i.e., change its shape and appearance. Once the force is removed the deformation is regarded - depending on the object aspect - as plastic, elastic, or elasto-plastic. A plastic deformation is permanent: the object maintains the shape caused by the force even after the force is removed. Elastic deformation results in the object returning to its original shape once the force is removed. Finally, elasto-plastic deformation combines both: the object neither returns to its original shape nor re- 
mains entirely deformed. This characterization corresponds to the property known in rheology as viscoelasticity of materials. Viscoelasticity has inspired the pioneer robotics works (Wada et al., 2001; Shibata and Hirai, 2006; Higashimori et al., 2010), where the object is modeled as a mesh of spring-damper particles. These three papers are recommended for their tutorial value.

To handle deformable objects, roboticists must address many challenges.

First, since the applied forces determine the object shape, the physical interaction between robot and object are crucial. This pushes the difficulty beyond that of most robotic tasks, which can be addressed using kinematics alone (Waldron and Schmiedeler, 2008), and not dynamics.

A second difficulty comes from the task definition itself: while rigid manipulation problems break down to placing the object in a desired pose, here the robot should also regulate the object shape. From a control viewpoint, this leads to an under-actuated control system, since a limited - robot-dependent - number of control inputs must regulate an infinite number of degrees of freedom (instead of the 6 of rigid body pose control). Such difficulty is palpable in finite element models (such as the viscoelastic ones cited above): while providing an elegant solution to the forward problem (determining the shape given the applied forces), they are incapable of solving the inverse (finding the forces needed to obtain a desired shape). The inverse geometric problem is solvable if one disposes of an explicit model of the object shape, as in the work of (Roussel et al., 2015).

Obviously, to obtain a desired shape, the robot controller must be capable of estimating online the current shape. This leads to the third challenge: observability, i.e., proper estimation of the object state (i.e., shape), from sensor measurements. Currently, the best way to do so is to rely on vision (Borum et al., 2014), be it 2D or 3D. Yet, non-rigid visual tracking is very demanding. While visual features of rigid objects can be consistently detected and tracked by exploiting a prior 3D model, on non-rigid materials features change over time. Feedback from force and tactile sensors could be beneficial, although this also requires accurate deformation and contact models that map force/tactile signals to the corresponding displacements of the object surface. This mapping is often very complicated to obtain.

Finally, an open methodological research question is whether a general unique paradigm can address all types of flexible manipulation. Such ultimate solver would be equivalent - for non-rigid manipulation - to what inverse kinematics control is for the rigid problem. Yet, as shown by the non-exhaustive survey that follows, this is far from true, since current research is mainly driven by applications in a bottom-up fashion that is doomed to be specific.

\section{Key Research Findings}

This section reviews the literature on robotic manipulation of flexible objects. The interested reader can also refer to the recent rich survey (Sanchez et al., 2018). That article follows a taxonomy based on geometry with three categories: objects are considered linear (e.g., beams, elastic tubes, cables, ropes, strings), planar (e.g., paper, clothes, fabric, metal sheets) or solid (e.g., food products, sponges, plush toys). Instead, the present essay classifies the research works according to the topic/methodology:

- works that focus on shape estimation, 
- works where the object is manipulated using path planning techniques,

- works where the object is shaped using sensor-based feedback control.

Each topic is addressed in one of the following sections.

\subsection{Shape Estimation}

As mentioned in Section 2, one of the main challenges in flexible manipulation is proper estimation of the object shape from sensor data. While estimating deformations has been thoroughly studied within the computer vision community, the field of robotics imposes strong computational time constraints which do not apply to standard vision. To give an idea, closing the feedback loop of most robotic controllers requires the sensor data to be updated roughly every tenth of a second.

Before the advent of the Microsoft Kinect, which made 3D (RGB-D) vision easily accessible, researchers relied on stereo cameras to track the shape of a pile for scooping (Sarata et al., 2004) and the surface of an object to be grasped (Khalil et al., 2010). The shape deformation, estimated with vision, can also be mapped to force and position measurements of a robotic hand, as in (Cretu et al., 2012). The proposed mapping, obtained with a neural network, accurately captures and predicts the shape of the object, while the robot fingers apply forces on it. Similarly, the authors of (Frank et al., 2014) estimate object deformation parameters (Young's modulus and Poisson ratio) by relating applied forces and resulting surface deformations. To this end, they rely on a volumetric Finite Element Model (FEM). A FEM is also used in (Petit et al., 2017) for mesh-fitting, to track in real time the shape of a 3D textureless object (a pizza dough) which undergoes elastic deformations. RGB-D vision is the only sensor required in this work. In one of his following works, Petit presents a method for tracking multiple interacting deformable objects, by registering image and RGB-D point clouds on FEMs (Petit et al., 2018). The authors of (Li et al., 2019) learn a particle-based simulator for complex control tasks. Their simulator can adapt to new environments or to unknown dynamics within few observations.

These articles all indicate that proper shape estimation requires a 3D model of the object, to be modified at each iteration according to the sensor output, with a prediction/update paradigm typical of control observer theory. For optimal performance, the developer should achieve an appropriate trade-off between the accuracy of such model and the computational constraints mentioned above.

\subsection{Manipulation Planning}

Two major approaches for task execution have emerged in the robotics literature: motion planning and sensor-based control. The planning approach breaks the task into discrete subtasks which satisfy the system constraints and possibly optimize some aspect of the task/mission. Low-dimensional problems are generally solved with grid-based algorithms that overlay a grid on the robot's configuration space. This approach generally relies on a priori knowledge of the future robot and environment states over a time window. On the other hand, sensor-based control closes the perception-to-action loop and is more appropriate in dynamic, unknown environments. The most known example is image-based visual servoing, which relies 
on visual feedback to reactively control robot motion. This section reviews works which address flexible manipulation with motion planning, whereas the next section will focus on sensor-based control.

One of the first works on the assembly of deformable objects (Zheng et al., 1991) addresses the insertion of a beam into a hole, by planning a priori the trajectory of the robot tool. (Moll and Kavraki, 2006) rely on a sampling-based approach to deform flexible wires, subject to manipulation constraints: they compute paths among minimal energy configurations. (Wakamutsu and Hirai, 2004) present a novel approach to linear object deformation. They first model the deformation (flexure, torsion, and extension) of this kind of object, and then apply path planning to modify its shape. The optimal paths are the ones that minimize the object potential energy. (Pham and Pham, 2018) consider robotic manipulation of a chain that is rotating at constant angular speed around a fixed axis. Having proved that the chain's configuration space is homeomorphic to a two-dimensional surface embedded in $\mathbb{R}^{3}$, the authors devise a strategy for transiting between different rotation modes in a stable and controlled manner. (Pan and Manocha, 2018) plan the motion of arbitrarily-shaped volumetric deformable bodies through complex environments. To this end, they compute trajectories that satisfy the dynamic constraints using a two-stage learning method: a multitask controller parameterized using dynamic movement primitives (DMP) followed by a neural-network that selects the DMP for driving the body while avoiding obstacles in the environment.

With the recent progress in computational power, researchers have integrated FEM within their planning frameworks. For instance (Yoshida et al., 2015) address the insertion of a gasket by relying on FEM to simulate its deformation. They use a motion planning algorithm with various objective functions (e.g., collision avoidance and minimum deformation), to generate the plans for extending/deforming the gasket before inserting it. Similarly (Roussel and Taïx, 2014) incorporate a physics engine in their motion planning algorithm. This engine represents a linear object as a connection of nodes, with the links modeled using an FEM, and the state space defined by the positions and velocities of all nodes. The authors sample the control commands, to find the one that moves the object towards the goal state, according to this physics engine.

While the cited works rely on motion planning, others propose sensor-based feedback control for object shaping. The next section reviews these works.

\subsection{Sensor-based Shape Control}

In contrast with manipulation planning, which is generally realized in open loop, sensor-based control relies on feedback from sensed data to modify the object shape online. The most popular sensors for this are force transducers and cameras.

The authors of (Higashimori et al., 2010) present a two-step framework: first they estimate - using force sensing - the elastic parameters of a four-element model, and then - based on the object plastic response - they compute and apply the force required to obtain the desired shape. Recently, David Navarro-Alarcon has designed a novel visual servoing scheme that explicitly deals with elastic deformations (Navarro-Alarcon et al., 2013, 2014). By estimating online the mapping (i.e., the interaction matrix) relating tool velocity and optical flow, he can actively 
deform compliant objects. While the results are groundbreaking, the proposed controller is only stable locally, i.e., it cannot guarantee global convergence. Inspired by these works (Laranjeira et al., 2017) derive the interaction matrix needed to deform catenary shaped objects (e.g., tethers or slack ropes). In (Schenck et al., 2017), the vision to action mapping is derived using a Convolutional Neural Network. The target application is scooping and dumping granular materials. The authors of (Cherubini et al., 2018) also rely on a neural network for mapping vision to action in a similar application. Yet, they train their network on data from human users. (Berenson, 2013) uses the concept of diminishing rigidity to compute an approximate Jacobian of the deformable object. In his work, the robot helps humans manipulate clothes.

\section{Future Directions for Research}

While great progress has been made, robotic manipulation of flexible objects is still at its early stages of development. Some of the main challenges have been indicated in Sect. 2. This last section suggests other possible avenues of future research.

As outlined above, both planning and feedback control approaches have been experimented. Historically, these two schools are disjoint within the robotics community, and unfortunately the same applies within the field of flexible manipulation. Yet, merging planning and feedback control methods would provide tremendous progress, by guaranteeing global convergence (through planning) albeit maintaining (through feedback control) adaptability to perturbations and unforeseen events. A very promising work in this direction is (McConachie et al., 2017). The authors propose a global planner that generates gross motion of the deformable object, along with a local controller that refines its configuration. A deadlock prediction algorithm determines when to use planning and when to use control.

Focusing on feedback control (Sect. 3.3) the reader will also notice that force and vision are seldom used within the same framework. Yet, these senses are complimentary and should be integrated; two preliminary works which do so are (Arriola-Rios and Wyatt, 2017) and (Ficuciello et al., 2018). In (Arriola-Rios and Wyatt, 2017), the object behavior is predicted by first classifying the material and then using force and computer vision to estimate its plastic and elastic deformations. The authors of (Ficuciello et al., 2018) identify the elasticity parameters of the object model using an RGB-D vision system coupled with a force sensor.

Despite the recent emergence of tactile sensing on robots (e.g., on robotic hand fingertips), very few researchers have exploited them for flexible manipulation. An exception is the work (Delgado et al., 2017), which presents an adaptable tactile-servo controller for in-hand manipulation of deformable objects. Here, tactile control consists in maintaining a given force value at the contact points. The value changes according to the object softness, which is estimated in an initial recognition stage.

Finally, to constrain the object's infinite degrees of freedom, the robot should make use of the environment contacts, as proposed by (Roussel et al., 2019) and by (Zhu et al., 2020). Dual arm robotic systems can also be used to this end. For example, the authors of (Kosuge et al., 1995) design a strategy for dual manipulation of a flexible metal sheet, based on force control. (Long et al., 2015) propose 
another approach for modeling the dynamics of two manipulators handling deformable objects. Here, the closed kinematic chain is divided into two subsystems based on their characteristics, i.e., the closed kinematic chain is decomposed into one flexible system (for the object) and one rigid system (for the manipulators). In (Zhu et al., 2018), a dual arm robot relies on visual servoing to change the shape of flexible cables.

In conclusion, as this essay has shown, flexible object manipulation will become paramount with the emergence of service robots. Although important results have been obtained, research in this field is still exploratory, promising to keep roboticists busy for the upcoming years.

\section{Conflict of interest}

The author declares that he has no conflict of interest.

\section{References}

Arriola-Rios VE, Wyatt JL (2017) A multi-modal model of object deformation under robotic pushing. IEEE Transactions on Cognitive and Developmental Systems 9(2):153-169

Berenson D (2013) Manipulation of deformable objects without modeling and simulating deformation. In: IEEE/RSJ Int. Conf. on Robots and Intelligent Systems, IROS

Borum A, Matthews D, Bretl T (2014) State estimation and tracking of deforming planar elastic rods. In: IEEE Int. Conf. on Robotics and Automation, ICRA

Cambridge Dictionary (2019) https://dictionary.cambridge.org

Cherubini A (2017) Model-free vision-based shaping of deformable plastic materials. https://www.lirmm.fr/lirmm_eng/research/teams/idh/flexbot

Cherubini A, Leitner J, Ortenzi V, Corke P (2018) Towards vision-based manipulation of plastic materials. In: IEEE/RSJ Int. Conf. on Robots and Intelligent Systems, IROS

Cretu AM, Payeur P, Petriu EM (2012) Soft Object Deformation Monitoring and Learning for Model-Based Robotic Hand Manipulation. IEEE Trans on Systems, Man and Cybernetics 42(3):740-753

Delgado A, Jara CA, Torres F (2017) In-hand recognition and manipulation of elastic objects using a servo-tactile control strategy. Robotics and ComputerIntegrated Manufacturing 48:102 - 112

Ficuciello F, Migliozzi A, Coevoet E, Petit A, Duriez C (2018) FEM-based deformation control for dexterous manipulation of 3D soft objects. In: IEEE/RSJ Int. Conf. on Robots and Intelligent Systems, IROS

Frank B, Stachniss C, Schmedding R, Teschner M, Burgard W (2014) Learning object deformation models for robot motion planning. Robotics and Autonomous Systems 62(8):1153-1174

Higashimori M, Yoshimoto K, Kaneko M (2010) Active shaping of an unknown rheological object based on deformation decomposition into elasticity and plasticity. In: IEEE Int. Conf. on Robotics and Automation, ICRA 
Khalil FF, Curtis P, Payeur P (2010) Visual monitoring of surface deformations on objects manipulated with a robotic hand. In: 2010 IEEE International Workshop on Robotic and Sensors Environments (ROSE)

Kosuge K, Yoshida H, Fukuda T, Sakai M, Kanitani K (1995) Manipulation of a flexible object by dual manipulators. In: IEEE Int. Conf. on Robotics and Automation, ICRA

Laranjeira M, Dune C, Hugel V (2017) Catenary-based visual servoing for tethered robots. In: IEEE Int. Conf. on Robotics and Automation, ICRA

Li Y, Wu J, Tedrake R, Tenenbaum J, Torralba A (2019) Learning particle dynamics for manipulating rigid bodies, deformable objects, and fluids. In: International Conference on Learning Representations, ICLR

Long P, Khalil W, Martinet P (2015) Dynamic modeling of cooperative robots holding flexible objects. In: Int. Conf. on Advanced Robotics, ICAR

Market Research Engine (2018) Service Robotics Market By Operating Environment Analysis; by Application Analysis and By Regional Analysis Global Forecast by 2018 - 2024. www.marketresearchengine.com/global-service-roboticsmarket

McConachie D, Ruan M, Berenson D (2017) Interleaving planning and control for deformable object manipulation. In: International Symposium on Robotics Research (ISRR)

Moll M, Kavraki LE (2006) Path planning for deformable linear objects. IEEE Trans on Robotics 22(4):625-636

Navarro-Alarcon D, Liu YH, Romero JG, Li P (2013) Model-free visually servoed deformation control of elastic objects by robot manipulators. IEEE Trans on Robotics 29(6):1457-1468

Navarro-Alarcon D, Liu Y, Romero JG, Li P (2014) On the visual deformation servoing of compliant objects: Uncalibrated control methods and experiments. Int Journal of Robotics Research 33(11)

Pan Z, Manocha D (2018) Realtime Planning for High-DOF Deformable Bodies Using Two-Stage Learning. In: IEEE Int. Conf. on Robotics and Automation, ICRA

Petit A, Lippiello V, Fontanelli GA, Siciliano B (2017) Tracking elastic deformable objects with an RGB-D sensor for a pizza chef robot. Robotics and Autonomous Systems 88:187-201

Petit A, Cotin S, Lippiello V, Siciliano B (2018) Capturing deformations of interacting non-rigid objects using RGB-D data. In: IEEE/RSJ Int. Conf. on Robots and Intelligent Systems, IROS

Pham H, Pham QC (2018) Robotic manipulation of a rotating chain. IEEE Trans on Robotics 34(1):139-150

Roussel O, Taïx M (2014) Deformable linear object manipulation planning with contacts. In: IEEE/RSJ Int. Conf. on Robots and Intelligent Systems, IROS

Roussel O, Renaud M, Taïx M (2015) Closed-forms of planar kirchhoff elastic rods: application to inverse geometry. In: IMA Conference on Mathematics of Robotics

Roussel O, Fernbach P, Taïx M (2019) Motion planning for an elastic rod using contacts. IEEE Trans on Automation Science and Engineering pp 1-14

Sanchez J, Corrales JA, Bouzgarrou BC, Mezouar Y (2018) Robotic manipulation and sensing of deformable objects in domestic and industrial applications: a survey. Int Journal of Robotics Research 37(7):688-716 
Sarata S, Osumi H, Kawai Y, Tomita F (2004) Trajectory arrangement based on resistance force and shape of pile at scooping motion. In: IEEE Int. Conf. on Robotics and Automation, ICRA

Schenck C, Tompson J, Fox D, Levine S (2017) Learning robotic manipulation of granular media. In: Conference on Robot Learning, CoRL

Shibata M, Hirai S (2006) Soft object manipulation by simultaneous control of motion and deformation. In: IEEE Int. Conf. on Robotics and Automation, ICRA, pp 2460-2465

Wada T, Hirai S, Kawamura S, Kamiji N (2001) Robust manipulation of deformable objects by a simple PID feedback. In: IEEE Int. Conf. on Robotics and Automation, ICRA, vol 1, pp 85-90 vol.1

Wakamutsu H, Hirai S (2004) Static modeling of linear object deformation based on differential geometry. Int Journal of Robotics Research 23(3):293-311

Waldron K, Schmiedeler J (2008) Kinematics. Springer Handbook of Robotics, Bruno Siciliano and Oussama Khatib Editors pp 9-33

Yoshida E, Ayusawa K, Ramirez-Alpizar I, Harada K, Duriez C, Kheddar A (2015) Simulation-based optimal motion planning for deformable object. In: 2015 IEEE International Workshop on Advanced Robotics and its Social Impacts

Zheng Y, Pei R, Chen C (1991) Strategies for automatic assembly of deformable objects. In: IEEE Int. Conf. on Robotics and Automation, ICRA

Zhu J, Navarro B, Fraisse P, Crosnier A, Cherubini A (2018) Dual-arm robotic manipulation of flexible cables. In: IEEE/RSJ Int. Conf. on Robots and Intelligent Systems, IROS

Zhu J, Navarro B, Passama R, Fraisse P, Crosnier A, Cherubini A (2020) Dual-arm robotic manipulation of deformable linear objects with environmental contacts. IEEE Robotics and Automation Letters 5(1):16-23 